\title{
Reflection of plane waves from free surface of a microstretch elastic solid
}

\author{
BALJEeT Singh \\ Department of Mathematics, Jat College, Rohtak 124 001, Haryana, India. \\ e-mail: baljeet_gill@hotmail.com
}

In the present investigation, it is shown that there exists five basic waves in a microstretch elastic solid half-space. The problem of reflection of plane waves from free surface of a microstretch elastic solid half-space is studied. The energy ratios for various reflected waves are obtained for aluminiumepoxy composite as a microstretch elastic solid half-space. The variations of the energy ratios with the angle of incidence are shown graphically. The microstretch effect is shown on various reflected waves.

\section{Introduction}

The linear theory of elasticity is of paramount importance in the stress analysis of steel, which is the commonest engineering structural material. To a lesser extent linear elasticity describes the mechanical behaviour of the other common solid materials, e.g., concrete, wood and coal. However, the linear theory of elasticity is unable to explain the behaviour of many of the new synthetic materials of the elastomer and polymer type, e.g., polymethyl-methacrylate (Perspex), polyethylene, polyvinyl chloride. The theory of micropolar elasticity is adequate to represent the behaviour of such materials. For ultrasonic waves i.e., for the case of elastic vibrations characterized by high frequencies and small wavelengths, the influence of the body microstructure becomes significant. This influence of microstructure results in the development of new type of waves, not found in the classical theory of elasticity.

Microstretch continuum is a model for Bravias lattice with a basis on the atomic level and a two phase dipolar solid with a core on the macroscopic level. For example, composite materials reinforced with chopped elastic fibres, porous media whose pores are filled with gas or invis- cid liquid, asphalt or other elastic inclusions and 'solid-liquid' crystals, etc., should be characterizable by microstretch solids. Eringen (1971, 1990) developed a theory of microstretch elastic solid in which he included microstructural expansions and contractions.

The problem of reflection of plane waves at the free surface of an elastic material is solved by Jeffreys (1926); Gutenberg (1944); Ewing et al (1957), etc.

The exact nature of the layers under the earth surface are not known. For the purpose of theoretical investigation about the earth's interior, we consider various appropriate models. The problems of waves and vibrations become more important in the field of seismology, when one studies the problem with additional parameters (e.g., microstretch, thermal disturbance, microrotation, porosity, viscosity, etc.). In the present paper, the basic equations of motion for microstretch elastic solid are solved and it is shown that there exist five basic waves in a microstretch elastic solid. The reflection of plane waves from free surface of a microstretch elastic solid halfspace is studied. The energy ratios of various reflected waves are computed and shown graphically with the angle of incidence of the striking wave.

Keywords. Microstretch elastic solid; reflection; energy ratios; aluminium-epoxy composite. 


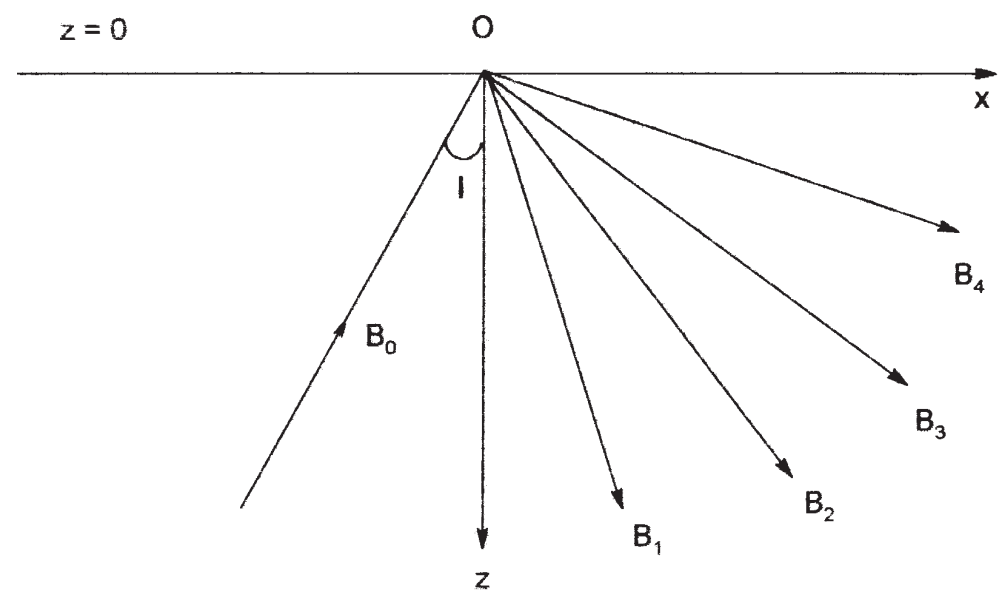

Figure 1. Geometry of the problem.

\section{Formulation of the problem}

Coordinate axes and the geometry of the wave system are defined with reference to figure 1 . The $x$ axis is taken along the free surface whereas the $z$ axis is taken normal to free surface in downward direction. The region $z>0$ is occupied by linear isotropic microstretch elastic solid. The $\mathrm{x}$ axis coincides with the direction of propagation of the surface wave. The plane of propagation of the incident wave is taken as the $x$ - $z$ plane.

Following Eringen (1990), the constitutive equations and field equations for linear isotropic microstretch elastic solid in the absence of body forces and body couples can be written as

$$
\begin{aligned}
t_{k l}= & \lambda u_{r, r} \delta_{k l}+\mu\left(u_{k, l}+u_{l, k}\right)+\kappa\left(u_{l, k}-\varepsilon_{k l r} \boldsymbol{\phi}_{r}\right) \\
& +\lambda_{o} \psi \delta_{k l} \\
m_{k l}= & \alpha \boldsymbol{\phi}_{r, r} \delta_{k l}+\beta \boldsymbol{\phi}_{k, l}+\gamma \boldsymbol{\phi}_{l, k} \\
\beta_{k}= & \alpha_{0} \psi_{, k}
\end{aligned}
$$

and

$$
\begin{aligned}
& \left(c_{1}^{2}+c_{3}^{2}\right) \nabla(\nabla \cdot \mathbf{u})-\left(c_{2}^{2}+c_{3}^{2}\right) \nabla \times(\nabla \times \mathbf{u}) \\
& \quad+c_{3}^{2} \nabla \times \boldsymbol{\phi}+\bar{\lambda}_{o} \nabla \psi=\ddot{\mathbf{u}}, \\
& \left(c_{4}^{2}+c_{5}^{2}\right) \nabla(\nabla \cdot \boldsymbol{\phi})-c_{4}^{2} \nabla \times(\nabla \times \boldsymbol{\phi})+\omega_{o}^{2} \nabla \\
& \quad \times \mathbf{u}-2 \omega_{o}^{2} \boldsymbol{\phi}=\ddot{\boldsymbol{\phi}}, \\
& c_{6}^{2} \nabla^{2} \psi-c_{7}^{2} \psi-c_{8}^{2}(\nabla \cdot \mathbf{u})=\ddot{\psi},
\end{aligned}
$$

where

$$
\begin{aligned}
& c_{1}^{2}=(\lambda+2 \mu) / \rho, c_{2}^{2}=\mu / \rho, \quad c_{3}^{2}=\kappa / \rho, \\
& c_{4}^{2}=\gamma / \rho j, \quad c_{5}^{2}=(\alpha+\beta) / \rho j, \omega_{0}^{2}=c_{3}^{2} / j=\kappa / \rho j, \\
& c_{6}^{2}=2 \alpha_{0} / 3 \rho j, \quad c_{7}^{2}=2 \lambda_{1} / 9 \rho j, \quad c_{8}^{2}=2 \lambda_{0} / 9 \rho j, \\
& \bar{\lambda}_{0}=\lambda_{0} / \rho
\end{aligned}
$$

Superposed dots on the right hand side of above equations denote the second partial derivative with respect to time, and

$$
\nabla=\frac{\partial}{\partial x} \hat{i}+\frac{\partial}{\partial y} \hat{j}+\frac{\partial}{\partial z} \hat{k} .
$$

By Helmholtz representation of vectors, we can write

$$
\begin{array}{rlrl}
\mathbf{u} & =\nabla q+\nabla \times \mathbf{U}, & \nabla . \mathbf{U}=\mathbf{0}, \\
\mathbf{\phi}=\nabla \xi+\nabla \times \boldsymbol{\Phi}, & \nabla . \boldsymbol{\Phi}=\mathbf{0} .
\end{array}
$$

Using equations (9) and (10), equations (4) to (6) reduce as

$$
\begin{aligned}
\left(c_{1}^{2}+c_{3}^{2}\right) \nabla^{2} q+\bar{\lambda}_{0} \psi & =\ddot{q}, \\
c_{6}^{2} \nabla^{2} \psi-c_{7}^{2} \psi-c_{8}^{2} \nabla^{2} q & =\ddot{\psi}, \\
\left(c_{2}^{2}+c_{3}^{2}\right) \nabla^{2} \mathbf{U}+c_{3}^{2} \nabla \times \boldsymbol{\Phi} & =\ddot{\mathbf{U}}, \\
c_{4}^{2} \nabla^{2} \boldsymbol{\Phi}-2 \omega_{0}^{2} \boldsymbol{\Phi}+\omega_{0}^{2} \nabla x \mathbf{U} & =\ddot{\boldsymbol{\Phi}}, \\
\left(c_{4}^{2}+c_{5}^{2}\right) \nabla^{2} \xi-2 \omega_{0}^{2} \xi & =\ddot{\xi} .
\end{aligned}
$$

Here, the equations (11) and (12) are coupled in $q$ and $\psi$ whereas the equations (13) and (14) are coupled in $\mathbf{U}$ and $\boldsymbol{\Phi}$. The equation (15) is uncoupled.

For two-dimensional motion in $x z$-plane, we take the displacement and microrotation vectors as

$$
\mathbf{u}=\left(u_{1}, 0, u_{3}\right) \text { and } \boldsymbol{\phi}=\left(0, \boldsymbol{\phi}_{2}, 0\right),
$$

where

$$
u_{1}=\frac{\partial q}{\partial x}-\frac{\partial U_{2}}{\partial z}, \quad u_{3}=\frac{\partial q}{\partial z}+\frac{\partial U_{2}}{\partial x} .
$$

For two-dimensional motion in $x z$-plane, we assume solutions $q$ and $\psi$ as

$$
\begin{aligned}
q & =\bar{q}(z) \exp [i(\omega t-k x)], \\
\psi & =\bar{\psi}(z) \exp [i(\omega t-k x)] .
\end{aligned}
$$


Substituting $q$ and $\psi$ given by equation (18) in equations (11) and (12) and then eliminating $q$ and $\psi$, we obtain

$$
\left(\nabla^{4}+A \nabla^{2}+B\right)(\bar{q}, \bar{\psi})=0
$$

where

$$
\begin{aligned}
& A=\left(\omega^{2} / V_{1}^{2}\right)+\left(\omega^{2}-c_{7}^{2}-a_{0} c_{8}^{2}\right) / c_{6}^{2}, \\
& B=\left(\omega^{2} / V_{1}^{2}\right)\left(\omega^{2}-c_{7}^{2}\right) / c_{6}^{2}
\end{aligned}
$$

and

$$
\begin{aligned}
V_{1}^{2}= & c_{1}^{2}+c_{3}^{2}, \quad a_{0}=\bar{\lambda}_{0} / V_{1}^{2}, \quad \nabla^{2}=-k^{2} \\
& +\left(d^{2} / d z^{2}\right) .
\end{aligned}
$$

The solution of equation (19) can be written as

$$
\bar{q}=q^{\prime}+q^{\prime \prime},
$$

where $q^{\prime}$ and $q^{\prime \prime}$ satisfy

$$
\begin{gathered}
\left(\nabla^{2}+\delta_{1}^{2}\right) q^{\prime}=0 \\
\left(\nabla^{2}+\delta_{2}^{2}\right) q^{\prime \prime}=0
\end{gathered}
$$

where

$$
\begin{aligned}
& \delta_{1}^{2}=(1 / 2)\left[A+\left(A^{2}-4 B\right)^{1 / 2}\right] \\
& \delta_{2}^{2}=(1 / 2)\left[A-\left(A^{2}-4 B\right)^{1 / 2}\right] .
\end{aligned}
$$

Then, the solution of the equation (19) can be written in the form

$$
\begin{aligned}
\bar{q}= & {\left[A_{1} \exp \left(m_{1} z\right)+A_{2} \exp \left(-m_{1} z\right)\right.} \\
& \left.+A_{3} \exp \left(m_{2} z\right)+A_{4} \exp \left(-m_{2} z\right)\right],
\end{aligned}
$$

where

$$
m_{1}^{2}=k^{2}-\delta_{1}^{2}, \quad m_{2}^{2}=k^{2}-\delta_{2}^{2},
$$

correspond to longitudinal displacement wave (LD-wave) and longitudinal microstretch wave (LMS-wave) respectively and $A_{1}, A_{2}, A_{3}, A_{4}$ are arbitrary constants. If we denote $V_{1}^{*}$ and $V_{2}^{*}$ as velocities of LD-wave and LMS-wave respectively, then

$$
V_{1}^{*}=\omega / \delta_{1}, \quad V_{2}^{*}=\omega / \delta_{2} .
$$

Taking second components of equation (13) and (14), we get

$$
\begin{aligned}
& \boldsymbol{\phi}_{2}=\frac{1}{c_{3}^{2}} \frac{\partial^{2} U_{2}}{\partial t^{2}}-\left(\frac{c_{2}^{2}}{c_{3}^{2}}+1\right) \nabla^{2} U_{2}, \\
& \boldsymbol{\phi}_{2}=\left(\omega_{0}^{2} \nabla^{2} U_{2}\right) /\left(c_{4}^{2} \nabla^{2}-2 \omega_{0}^{2}-\frac{\partial^{2}}{\partial t^{2}}\right) .
\end{aligned}
$$

Eliminating $\boldsymbol{\phi}_{2}$ from equations (26) and (27) and assuming $U_{2}$ as

$$
U_{2}=f(z) \exp \{i(\omega t-k x)\},
$$

we obtain the following linear differential equation,

$$
\left(\frac{d^{4}}{d z^{4}}+C \frac{d^{2}}{d z^{2}}+D\right) f(z)=0
$$

where

$$
\begin{aligned}
C=k^{2} & \left(\frac{c^{2}}{c_{2}^{2}+c_{3}^{2}}+\frac{c^{2}}{c_{4}^{2}}-2\right)+\frac{\omega_{0}^{2}}{c_{4}^{2}}\left(\frac{c_{3}^{2}}{c_{2}^{2}+c_{3}^{2}}-2\right), \\
D= & k^{4}\left(1-\frac{c^{2}}{c_{2}^{2}+c_{3}^{2}}-\frac{c^{2}}{c_{4}^{2}}+\frac{c^{4}}{c_{4}^{2}\left(c_{2}^{2}+c_{3}^{2}\right)}\right) \\
& -k^{2}\left\{\frac{\omega_{0}^{2}}{c_{4}^{2}}\left(\frac{c_{3}^{2}}{c_{2}^{2}+c_{3}^{2}}+\frac{2 c^{2}}{c_{2}^{2}+c_{3}^{2}}-2\right) .\right.
\end{aligned}
$$

The solution of equation (29) can be written as

$$
\begin{aligned}
f(z)= & A_{5} \exp \left(m_{3} z\right)+A_{6} \exp \left(-m_{3} z\right) \\
& +A_{7} \exp \left(m_{4} z\right)+A_{8} \exp \left(-m_{4} z\right)
\end{aligned}
$$

where

$$
\begin{aligned}
& m_{3}=\left\{(1 / 2)\left[\left(C^{2}-4 D\right)^{1 / 2}-C\right]\right\}^{1 / 2} \text { and } \\
& m_{4}=\left\{(-1 / 2)\left[\left(C^{2}-4 D\right)^{1 / 2}+C\right]\right\}^{1 / 2}
\end{aligned}
$$

correspond to coupled transverse microrotational waves and coupled transverse displacement waves (CD I- and CD II-waves) and $A_{5}, A_{6}, A_{7}, A_{8}$ are arbitrary constants. If we denote $V_{3}^{*}$ and $V_{4}^{*}$ as velocities of CDI and CD II waves respectively, then

$$
V_{3}^{* 2}=\omega^{2} /\left(k^{2}-m_{3}^{2}\right), \quad V_{4}^{* 2}=\omega^{2} /\left(k^{2}-m_{4}^{2}\right) .
$$

From equations (28) and (32), we obtain

$$
\begin{aligned}
U_{2}=\{ & A_{5} \exp \left(m_{3} z\right)+A_{6} \exp \left(-m_{3} z\right)+A_{7} \exp \left(m_{4} z\right) \\
& \left.+A_{8} \exp \left(-m_{4} z\right)\right\} \exp \{i(\omega t-k x)\} .
\end{aligned}
$$

The solution of the equation (15) corresponds to the longitudinal microrotational wave (LM-wave) as obtained by Parfitt and Eringen (1969).

Thus, there exists five basic waves in a linear isotropic microstretch elastic solid half-space. 


\section{Wave motions}

For two-dimensional motion, the boundary conditions at the free surface $z=0$, are the vanishing of normal and tangential force stresses, tangential couple stress, and vector first moment at the free surface $z=0$; that is

$$
t_{z z}=0, \quad t_{z x}=0, \quad m_{z y}=0, \quad \beta_{z}=0 .
$$

Corresponding to incident plane waves (LD or CD II wave), four waves (LD-wave, LMS-wave, two sets of two coupled waves CD I-, CD II- waves) will propagate in microstretch elastic solid half-space after reflection from free surface $z=0$. For an incident longitudinal displacement wave (LD wave), $c=V_{1}^{*} \operatorname{cosec}$ I whereas $c=V_{4}^{*} \operatorname{cosec} \mathrm{I}$, for an incident coupled transverse displacement wave (CD II wave), where I is the angle of incidence.

In a microstretch elastic solid, the appropriate potential functions for incident and reflected waves are as follows

$$
\begin{aligned}
q=\{ & B_{0} \exp \left(m_{1} z\right)+B_{1} \exp \left(-m_{1} z\right) \\
& \left.+B_{2} \exp \left(-m_{2} z\right)\right\} \exp [i(\omega t-k x)], \\
\psi= & \zeta_{1} B_{0} \exp \left(m_{1} z\right)+\zeta_{1} B_{1} \exp \left(-m_{1} z\right) \\
& \left.+\zeta_{2} B_{2} \exp \left(-m_{2} z\right)\right\} \exp [i(\omega t-k x)], \\
U_{2}=\{ & B_{0} \exp \left(m_{4} z\right)+B_{3} \exp \left(-m_{3} z\right) \\
& \left.+B_{4} \exp \left(-m_{4} z\right)\right\} \exp [i(\omega t-k x)], \\
\phi_{2}= & \eta_{1} B_{0} \exp \left(m_{4} z\right)+\eta_{2} B_{3} \exp \left(-m_{3} z\right) \\
& \left.+\eta_{2} B_{4} \exp \left(-m_{4} z\right)\right\} \exp [i(\omega t-k x)],
\end{aligned}
$$

where

$$
\begin{aligned}
\zeta_{1,2}= & \left\{k^{2}-m_{1,2}^{2}-\left(\omega^{2} / V_{1}^{2}\right)\right\} / a_{0}, \\
\eta_{1,2}= & k^{2}\left\{\left(1+\left(c_{2}^{2} / c_{3}^{2}\right)-\left(c^{2} / c_{3}^{2}\right)\right\}\right. \\
& -m_{3,4}^{2}\left\{\left(1+\left(c_{2}^{2} / c_{3}^{2}\right)\right\}\right.
\end{aligned}
$$

and for an incident LD wave, put $B_{0}=0$ in equations (38) and (39), whereas for an incident CD II wave, put $B_{0}=0$ in equations (36) and (37).

\section{Solution of the problem}

Making use of the potentials given by equations (36) to (39) in boundary conditions (35) after using the equations (1) to (3), (9), (10), (16) and (17), we obtain the following system of nonhomogeneous equations as

$$
\sum_{j=1}^{4} a_{i j} Z_{j}=b_{i}, \quad(i=1,2, ., 4),
$$

where

$$
\begin{aligned}
& a_{11}=\lambda\left(m_{1}^{2}+k^{2}\right)+(2 \mu+\kappa) m_{1}^{2}+\lambda_{0} \zeta_{1}, \\
& a_{12}=\lambda\left(m_{2}^{2}+k^{2}\right)+(2 \mu+\kappa) m_{2}^{2}+\lambda_{0} \zeta_{2}, \\
& a_{13}=i(2 \mu+\kappa) k m_{3}, \quad a_{14}=i(2 \mu+\kappa) k m_{4}, \\
& a_{21}=i(2 \mu+\kappa) k m_{1}, \quad a_{22}=i(2 \mu+\kappa) k m_{2}, \\
& a_{23}=-\left\{\mu\left(k^{2}+m_{3}^{2}\right)+\kappa\left(m_{3}^{2}+\eta_{1}\right)\right\}, \\
& a_{24}=-\left\{\mu\left(k^{2}+m_{4}^{2}\right)+\kappa\left(m_{4}^{2}+\eta_{2}\right)\right\}, \\
& a_{31}=a_{32}=0, \quad a_{33}=-m_{3} \eta_{1}, \quad a_{34}=-m_{4} \eta_{2}, \\
& a_{41}=-m_{1} \zeta_{1}, \quad a_{42}=-m_{2} \zeta_{2}, \quad a_{43}=a_{44}=0,
\end{aligned}
$$

and

(a) for an incident LD wave

$$
b_{1}=-a_{11}, \quad b_{2}=a_{21}, \quad b_{3}=a_{31}, \quad b_{4}=a_{41},
$$

(b) for an incident CD II wave

$$
b_{1}=a_{14}, \quad b_{2}=-a_{24}, \quad b_{3}=a_{34}, \quad b_{4}=-a_{44},
$$

and

$$
\begin{aligned}
Z_{1}= & B_{1} / B_{0}, \quad Z_{2}=B_{2} / B_{0}, \quad Z_{3}=B_{3} / B_{0}, \\
& Z_{4}=B_{4} / B_{0},
\end{aligned}
$$

are the amplitude ratios for reflected LD-wave, LMS-wave, CD I- and CD II-waves respectively.

We shall now consider the partitioning of energy between different reflected waves at a surface element of unit area. Following Achenbach (1973), the instantaneous rate of work of surface traction is the scalar product of the surface traction and the particle velocity. This scalar product is called the power per unit area, denoted by $P^{*}$, and represents the rate at which the energy is communicated per unit area of the surface, i.e., the energy flux across the surface element. The time average of $P^{*}$ over a period, denoted by $\left\langle P^{*}\right\rangle$, represents the average energy transmission per unit surface area per unit time. For the microstretch elastic solid, the rate of energy transmission at the free surface $z=0$ is given by

$$
P^{*}=t_{z z} \dot{u}_{3}+t_{z x} \dot{u}_{1}+m_{z y} \dot{\phi}_{2} .
$$

Following Achenbach (1973), for any two complex functions of the forms

$$
F=F_{o} e^{i\left(\omega t-\gamma_{1}\right)}, \quad f=f_{o} e^{i\left(\omega t-\gamma_{2}\right)},
$$

where $F_{o}$ and $f_{o}$ are real-valued, the following is relation for time average of a product of real parts of two complex functions $F$ and $f$

$$
\langle R(F) \times R(f)\rangle=R(F \bar{f}) / 2 .
$$




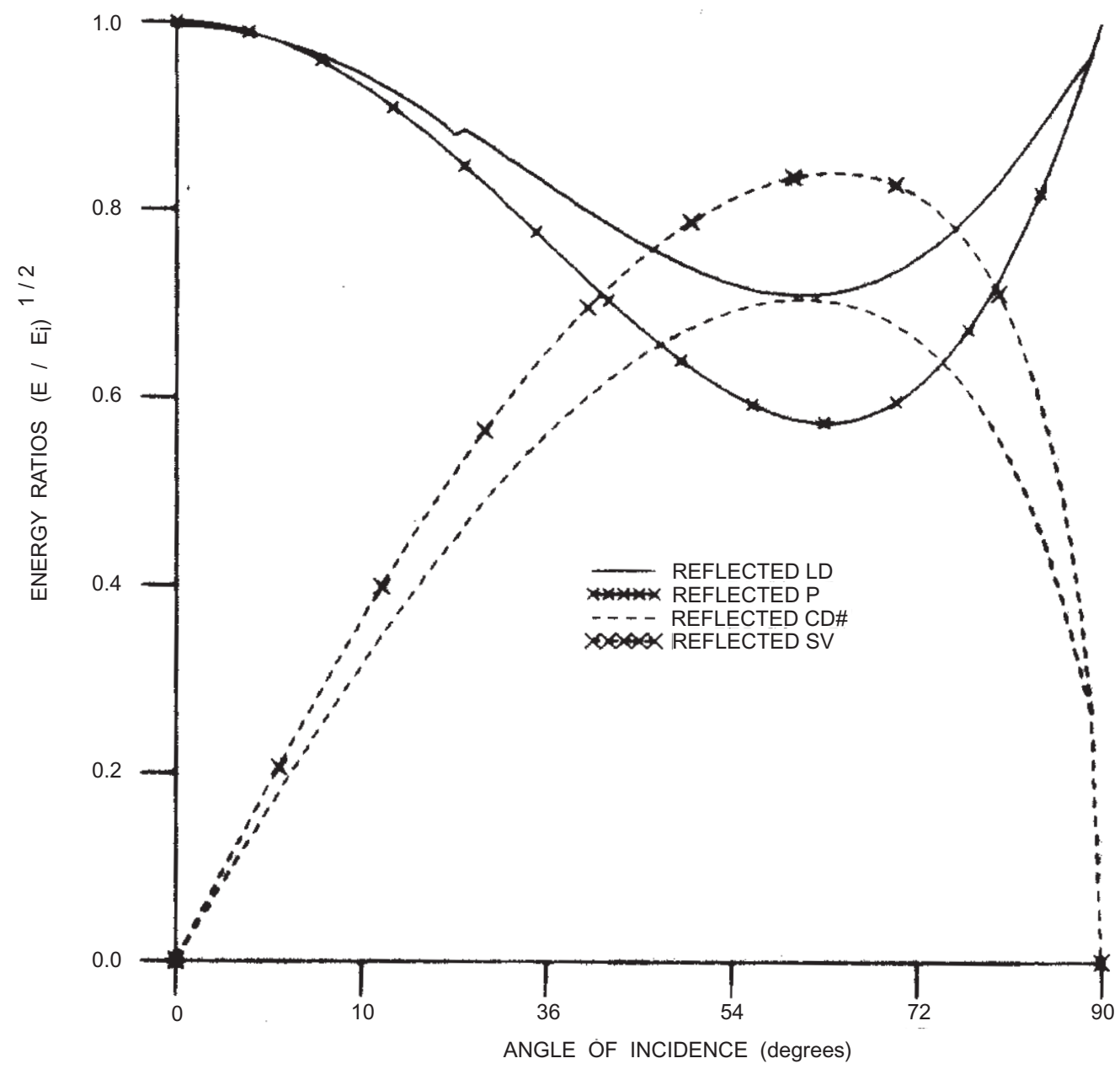

Figure 2. Variations of the square roots of the energy ratios with the angle of incidence of incident LD wave.

We shall now calculate $\left\langle P^{*}\right\rangle$ for the incident and each of the reflected wave using the appropriate displacement potentials and hence obtain the energy ratios giving the time rate of average energy transmission for the respective wave to that of the incident wave. The expressions for these energy ratios $E_{i}(i=1,2, ., 4)$ for the reflected LD, reflected LMS, reflected CD I and reflected CD II waves respectively are given by

$$
E_{i}=\left\langle P_{i}^{*}\right\rangle /\left\langle P_{o}^{*}\right\rangle, \quad(i=1,2, ., 4),
$$

where

$$
\begin{aligned}
\left\langle P_{1}^{*}\right\rangle=[ & {\left[(1 / 2)\left\{(\lambda+2 \mu+\kappa)\left(m_{1}^{2}-k^{2}\right)+m_{1} \lambda_{o} \zeta_{1}\right\}\right] } \\
& \left(m_{1} / m_{o}\right)\left|Z_{1}\right|^{2}, \\
\left\langle P_{2}^{*}\right\rangle= & {\left[(1 / 2)\left\{(\lambda+2 \mu+\kappa)\left(m_{2}^{2}-k^{2}\right)+m_{2} \lambda_{o} \zeta_{2}\right\}\right] } \\
& \left(m_{2} / m_{o}\right)\left|Z_{2}\right|^{2}, \\
\left\langle P_{3}^{*}\right\rangle= & {\left[(1 / 2)\left\{(\mu+\kappa)\left(m_{3}^{2}-k^{2}\right)+\gamma \eta_{1}^{2}+\kappa \eta_{1}\right\}\right] } \\
& \left(m_{3} / m_{o}\right)\left|Z_{3}\right|^{2},
\end{aligned}
$$

$$
\begin{aligned}
\left\langle P_{4}^{*}\right\rangle= & {\left[(1 / 2)\left\{(\mu+\kappa)\left(m_{4}^{2}-k^{2}\right)+\gamma \eta_{2}^{2}+\kappa \eta_{2}\right\}\right] } \\
& \left(m_{4} / m_{o}\right)\left|Z_{4}\right|^{2},
\end{aligned}
$$

and

(a) for an incident LD wave:

$$
\begin{aligned}
\left\langle P_{o}^{*}\right\rangle= & -\left[( 1 / 2 ) \left\{(\lambda+2 \mu+\kappa)\left(m_{1}^{2}-k^{2}\right)\right.\right. \\
& \left.\left.+m_{1} \lambda_{o} \zeta_{1}\right\}\right], \quad m_{o}=m_{1} .
\end{aligned}
$$

(b) for an incident CD II wave:

$$
\begin{aligned}
\left\langle P_{o}^{*}\right\rangle= & -\left[(1 / 2)\left\{(\mu+\kappa)\left(m_{4}^{2}-k^{2}\right)+\gamma \eta_{2}^{2}+\kappa \eta_{2}\right\}\right], \\
& m_{o}=m_{4} .
\end{aligned}
$$

\section{Numerical results and discussion}

Following Gauthier (1982), we take the case of aluminium-epoxy composite (microstretch elastic solid) for the purpose of numerical calculations. The physical constants used are given as 


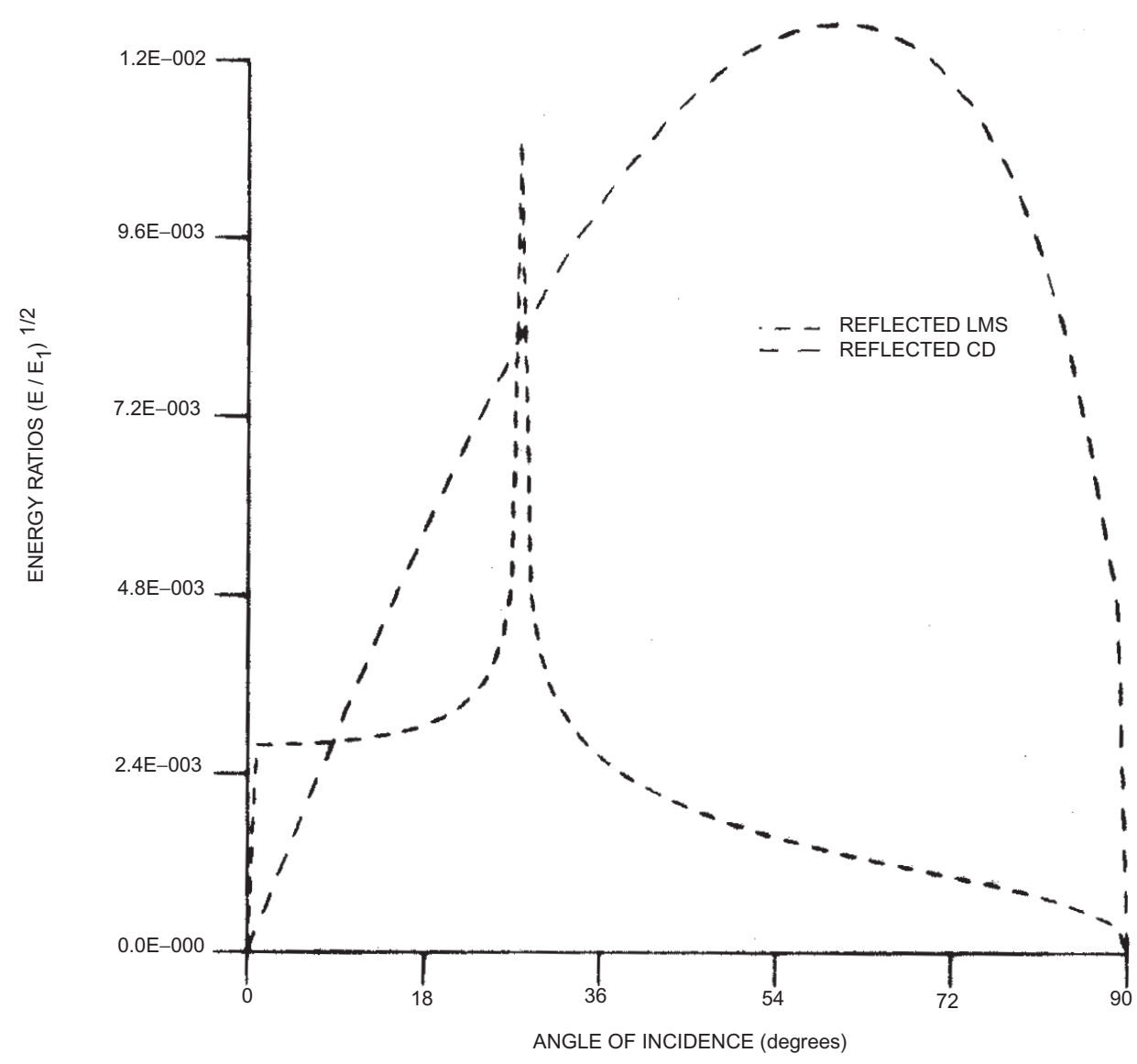

Figure 3. Variations of the energy ratios for reflected waves with the angle of incidence of incident LD wave.

$$
\begin{aligned}
& \gamma=7.59 \times 10^{11} \text { dyne } / \mathrm{cm}^{2}, \\
& \kappa=0.0149 \times 10^{11} \text { dyne } / \mathrm{cm}^{2}, \\
& \lambda_{o}=0.62 \times 10^{11} \text { dyne }, \\
& \alpha_{o}=1.45 \times 10^{11} \text { dyne } \\
& j=0.0196 \mathrm{~cm}^{2},
\end{aligned}
$$

For the above values of the relevant physical constants, the systems of equations (42) are solved for amplitude ratios by the application of the Gauss elimination method for each value of angle of incidence of the incident LD- (or CD II-) wave varying from normal incidence to grazing incidence. Corresponding values of the energy ratios depending upon the angle of incidence are computed using the relations (45) to (48). The variations of the square root energy ratios of reflected waves with the angle of incidence have been shown graphically in figures $2,3,4$ and 5 for incident LD- and CD II-waves respectively. The lines with centre symbols represent the variations for reflected $P$ - and $S V$-waves respectively after neglecting the microstretch effect (elastic case).

$$
\begin{aligned}
& \mu=1.89 \times 10^{11} \text { dyne } / \mathrm{cm}^{2}, \\
& \gamma=0.0268 \times 10^{11} \text { dyne }, \\
& \lambda_{1}=0.63 \times 10^{11} \text { dyne }, \\
& \rho=2.19 \mathrm{gm} / \mathrm{cm}^{3}, \\
& \omega^{2} / \omega_{o}^{2}=10 .
\end{aligned}
$$

\subsection{Incident LD-wave}

The variations of square root of energy ratio for reflected LD and CDII waves with the angle of incidence of the incident LD-wave have been shown by solid and dashed lines respectively in figure 2 . The square root of the energy ratio for reflected LD has its value one at $I=0^{\circ}$ and $I=90^{\circ}$ and attains its minima at $I=61^{\circ}$. Also, the square root of the energy ratio for reflected CD II has its value zero at $I=0^{\circ}$ and $I=90^{\circ}$ and attains its maxima at $I=61^{\circ}$. The solid and dashed lines with centre symbols shows the variations of reflected $P$ and reflected $S V$ waves respectively in figure 2 . A comparison between lines with and without centre symbols shows the microstretch effect on reflected elastic waves. 


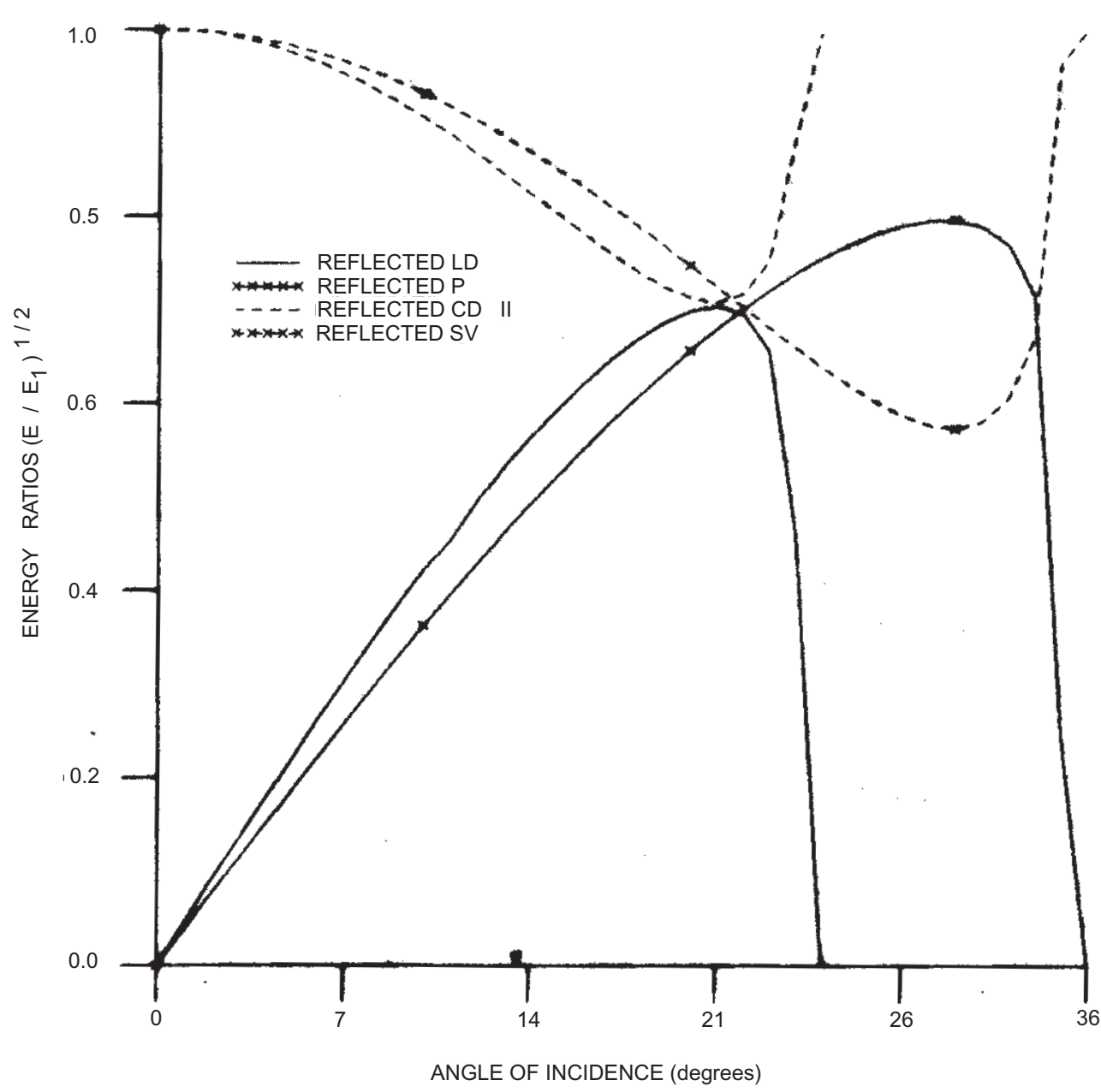

Figure 4. Variations of the energy ratios for reflected waves with the angle of incidence of incident CD II wave.

The variations of the square root of energy ratios for reflected LMS and CD I waves with the angle of incidence varying from normal to grazing incidence have been depicted in figure 3 . It is the microstretch effect due to which the above two new waves appear in a microstretch elastic solid.

The sum of energy ratios (normalized energy) at each angle of incidence for various reflected waves in a microstretch elastic solid is found to be equal to unity as in case of an elastic solid.

\subsection{Incident CD II-wave}

The variations of square root of energy ratio for reflected LD and CD II waves with the angle of incidence of the incident CD II-wave have been shown by solid and dashed lines respectively in figure 4 . The square root of the energy ratio for reflected LD has its value zero at $I=0^{\circ}$. It increases and attains its maxima near $I=21^{\circ}$. For the range $21^{\circ} \leq I \leq 24^{\circ}$, it decreases. Beyond $I=24^{\circ}$, it becomes zero at each angle of incidence. Also, the square root of the energy ratio for reflected CD II has its value one at $I=0^{\circ}$ and decreases to its minima at $I=21^{\circ}$. For the range $21^{\circ} \leq I \leq 24^{\circ}$, it increases. Beyond $I=24^{\circ}$, it becomes one at each angle of incidence. The solid and dashed lines with centre symbols shows the variations of reflected $P$ and reflected $S V$ waves respectively in figure 4 . A comparison between lines with and without centre symbols shows the microstretch effect on reflected elastic waves.

The variations of the square root of energy ratios for reflected LMS and CD I waves with the angle of incidence of incident CD II wave have been depicted in figure 5 . Beyond $I=24^{\circ}$, these waves disappear.

The sum of energy ratios (normalized energy) at each angle of incidence for various reflected waves in a microstretch elastic solid is also found to be equal to unity.

\section{Conclusions}

Detailed numerical calculations have been presented for the case of both LD- and CD II-waves incident at the free surface of a microstretch elastic 


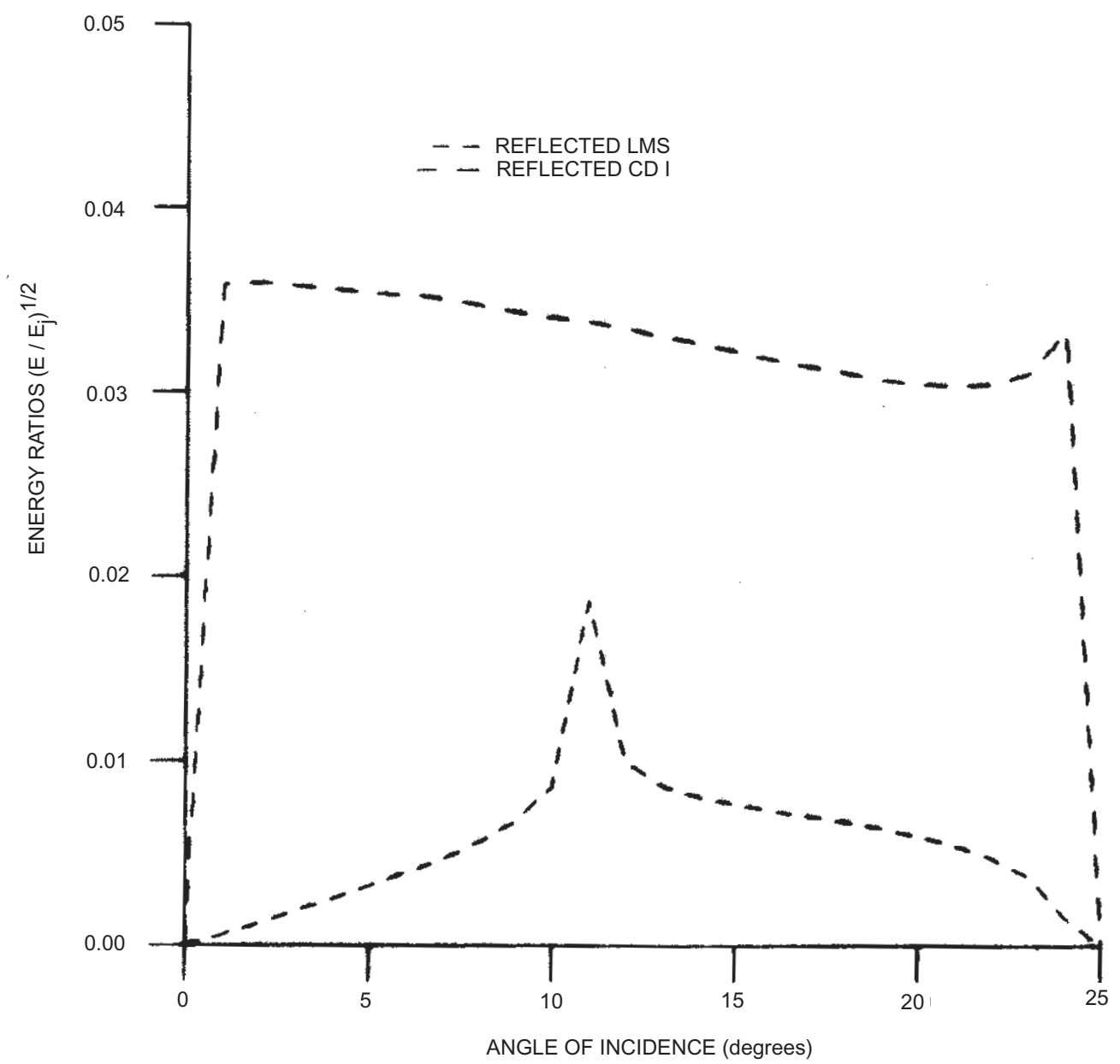

Figure 5. Variations of the energy ratios for reflected waves with the angle of incidence of incident CD II wave.

solid (aluminium-epoxy composite) and the results obtained agree fairly with those of Ewing et al (1957). If we neglect the microstretch effect, it is found that the LD and CD II waves become $P$ and $S V$ waves respectively whereas the LMS and CD I waves do not appear. In case of an incident CD II wave, the critical angle is found to be at $I=24^{\circ}$. After neglecting the microstretch, the critical angle shifts at $I=34^{\circ}$ for the case of an incident $S V$ wave. The normalized energy at each angle of incidence of incident LD (or CD II) wave is found to be equal to unity. Thus, the microstretch effect plays an important role in reflection phenomenon. The current problem is the more realistic model of the earth. Experimental seismologists can make use of this model for estimation of earthquake predictions.

\section{List of Symbols}

$\rho \quad$ the density of linear microstretch elastic solid.

$\mathbf{u}$ the displacement vectors.

$\phi \quad$ the microrotation vectors.

$\psi \quad$ the scalar microstretch quantity.

$\lambda, \mu$ the Lame's constants.

\begin{tabular}{|c|c|}
\hline$\alpha, \beta, \gamma, \kappa$ & the microrotation constants. \\
\hline$\alpha_{0}, \lambda_{0}, \lambda_{1}$ & the microstretch constants. \\
\hline$j$ & the microrotational inertia. \\
\hline$q, \xi$ & the scalar potentials. \\
\hline $\mathbf{U}, \Phi$ & the vector potentials. \\
\hline$\nabla$ & the del operator. \\
\hline$u_{1}, u_{2}, u_{3}$ & $\begin{array}{l}\text { the components of displacement } \\
\text { vector } \mathbf{u} \text {. }\end{array}$ \\
\hline$\phi_{1}, \phi_{2}, \phi_{3}$ & $\begin{array}{l}\text { the component of microrotation } \\
\text { vector } \phi \text {. }\end{array}$ \\
\hline$k$ & the wave number. \\
\hline$c$ & $\begin{array}{l}\text { apparent phase velocity on the } \\
\text { surface. }\end{array}$ \\
\hline$\omega(=k c)$ & angular frequency. \\
\hline$U_{1}, U_{2}, U_{3}$ & the components of vector $\mathbf{U}$ \\
\hline $\mathrm{R}(\mathrm{F}), \mathrm{R}(\mathrm{f})$ & $\begin{array}{l}\text { the real parts of complex functions } \\
\mathrm{F} \text { and } \mathrm{f} \text {. }\end{array}$ \\
\hline & the complex conjugate of function $f$. \\
\hline
\end{tabular}

\section{References}

Achenbach J D 1973 Wave propagation in elastic solids, (Amsterdam: North Holland) 
Eringen A C 1971 Micropolar elastic solids with stretch; Ari. Kitabevi. Matbassi. 24 1-9

Eringen A C 1990 Theory of thermo-microstretch elastic solid; Int. J. Engng. Sci., 28 1291-1296

Ewing W M, Jardetzky W S and Press F 1957 Elastic waves in layered media, (New York: McGraw-Hill)

Gauthier R D 1982 Experimental investigation on micropolar media, Mechanics of Micropolar Media (ed.) O Brulin and R K T Hsieh), (Singapore: World Scientific)
Gutenberg B 1944 Energy ratio of reflected and refracted seismic waves; Bull. Seism. Soc. Amer. 34 85-90

Jeffereys H 1926 The reflection and refraction of elastic waves, Mon. Not. R. Astro. Soc. Geophys. Supp. 1, 321326

Parfitt V R and Eringen A C 1969 Reflection of plane waves from flat boundary of a micropolar elastic half-space; $J$. Acoust. Soc. Am. 45 1258-1272

MS received 14 September 1999; revised 7 October 2001 